Direct monitoring of calcium-triggered phase transitions in cubosomes using smallangle X-ray scattering combined with microfluidics

Ghazal, Aghiad; Gontsarik, Mark; Kutter, Jörg P.; Lafleur, Josiane P.; Labrador, Ana;

Mortensen, Kell; Yaghmur, Anan

Published in:

Journal of Applied Crystallography

DOI:

10.1107/S1600576716014199

Publication date:

2016

Document version

Publisher's PDF, also known as Version of record

Citation for published version (APA):

Ghazal, A., Gontsarik, M., Kutter, J. P., Lafleur, J. P., Labrador, A., Mortensen, K., \& Yaghmur, A. (2016). Direct monitoring of calcium-triggered phase transitions in cubosomes using small-angle X-ray scattering combined with microfluidics. Journal of Applied Crystallography, 49(6), 2005-2014.

https://doi.org/10.1107/S1600576716014199 


\title{
Direct monitoring of calcium-triggered phase transitions in cubosomes using small-angle $X$-ray scattering combined with microfluidics
}

\author{
Aghiad Ghazal, Mark Gontsarik, Jörg P. Kutter, Josiane P. Lafleur, Ana \\ Labrador, Kell Mortensen and Anan Yaghmur
}

J. Appl. Cryst. (2016). 49, 2005-2014

\section{- IUCr Journals CRYSTALLOGRAPHY JOURNALS ONLINE}

Copyright (C) International Union of Crystallography

Author(s) of this paper may load this reprint on their own web site or institutional repository provided that this cover page is retained. Republication of this article or its storage in electronic databases other than as specified above is not permitted without prior permission in writing from the IUCr.

For further information see http://journals.iucr.org/services/authorrights.html 
JOURNAL OF

APPLIED

CRYSTALLOGRAPHY

ISSN 1600-5767

Received 3 May 2016

Accepted 6 September 2016

Edited by J. Hajdu, Uppsala University, Sweden

Keywords: lipidic formulations; microfabrication; microfluidics; small-angle X-ray scattering; SAXS; X-ray techniques.

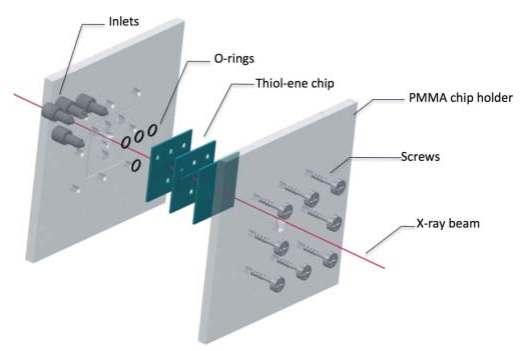

(C) 2016 International Union of Crystallography

\section{Direct monitoring of calcium-triggered phase transitions in cubosomes using small-angle X-ray scattering combined with microfluidics}

\author{
Aghiad Ghazal, ${ }^{\mathrm{a}, \mathrm{b}}$ Mark Gontsarik, ${ }^{\mathrm{b}}$ Jörg P. Kutter, ${ }^{\mathrm{b}}$ Josiane P. Lafleur, ${ }^{\mathrm{b}}$ Ana \\ Labrador, $^{\mathrm{c}}$ Kell Mortensen ${ }^{\mathrm{a}}$ and Anan Yaghmur ${ }^{\mathrm{b}}$ *
}

\footnotetext{
${ }^{\mathbf{a}}$ Niels Bohr Institute, University of Copenhagen, Copenhagen, Denmark, ${ }^{\mathbf{b}}$ Department of Pharmacy, University of Copenhagen, Copenhagen, Denmark, and ${ }^{\mathbf{C}}$ Max IV Laboratory, Lund University, Lund, Sweden. *Correspondence e-mail: anan.yaghmur@sund.ku.dk
}

This article introduces a simple microfluidic device that can be combined with synchrotron small-angle X-ray scattering (SAXS) for monitoring dynamic structural transitions. The microfluidic device is a thiol-ene-based system equipped with $125 \mu \mathrm{m}$-thick polystyrene windows, which are suitable for X-ray experiments. The device was prepared by soft lithography using elastomeric molds followed by a simple UV-initiated curing step to polymerize the chip material and simultaneously seal the device with the polystyrene windows. The microfluidic device was successfully used to explore the dynamics of the structural transitions of phytantriol/dioleoylphosphatidylglycerol-based cubosomes on exposure to a buffer containing calcium ions. The resulting SAXS data were resolved in the time frame between 0.5 and $5.5 \mathrm{~s}$, and a calcium-triggered structural transition from an internal inverted-type cubic phase of symmetry $\operatorname{Im} 3 m$ to an internal inverted-type cubic phase of symmetry Pn3m was detected. The combination of microfluidics with X-ray techniques opens the door to the investigation of early dynamic structural transitions, which is not possible with conventional techniques such as glass flow cells. The combination of microfluidics with X-ray techniques can be used for investigating protein unfolding, for monitoring the formation of nanoparticles in real time, and for other biomedical and pharmaceutical investigations.

\section{Introduction}

Advancements in micro- and nano-fabrication methods have made microfluidics an attractive platform for basic and applied research studies and various biomedical and pharmaceutical applications. These applications include protein crystallization (Hansen et al., 2002), high-throughput screening in drug development (Whitesides, 2006; Dittrich \& Manz, 2006), bioanalysis of drugs and bioactive materials (Sia \& Whitesides, 2003), manipulation of single cells and single molecules (Wheeler et al., 2003; Werdich et al., 2004; Dittrich \& Manz, 2005; Stavis et al., 2005), formation of monodispersed bubbles (Gañán-Calvo \& Gordillo, 2001), and nano-objects (Nie et al., 2008; Thorsen et al., 2001; Link et al., 2004; Anna et $a l ., 2003)$. In these applications, the main advantages are often related to the development of efficient miniaturized devices with a minimal sample consumption, which is of special interest when studying expensive materials. Such devices open up the possibility of maintaining a more precise control over the mixing environment compared to conventional methods of mixing, such as bulk mixing, and the possibility of performing a complete chemical analysis (Price \& Culbertson, 2007). An important aspect of miniaturized systems for chemical and biochemical applications is the possibility of monitoring 
reactions and assays for detecting the formation or the degradation of chemical substances in situ. A number of detection techniques have been associated with microfluidics; many of them are optical in nature, including fluorescence (Llobera et al., 2007; Edel et al., 2004), absorbance (Steigert et al., 2006), chemiluminescence (Marchand et al., 2008) and Raman spectroscopy (Liu et al., 2011). In addition, there is a growing interest in exploiting the benefits of combining microfluidics and X-ray techniques for different studies, including the dynamic mechanism of protein folding (Pollack et al., 1999), crystallization (Guha et al., 2012; Pawate et al., 2015), and nucleation and growth of nanoparticles (Polte et al., 2010).

X-ray techniques are powerful techniques for studying selfassembled nanostructures of various materials consisting of amphiphilic surfactant-like lipids or polymers (Azmi, Wu et al., 2015; Wibroe et al., 2015; Nilsson et al., 2013; Yaghmur \& Rappolt, 2012; Yaghmur, Larsen et al., 2011; Yaghmur \& Glatter, 2009; Dehsorkhi et al., 2014; Shrestha et al., 2015; Narayanan, 2009; Yaghmur et al., 2010; Azmi et al., 2016). Conventionally, small-angle X-ray (SAXS) and neutron scattering methods are the most widely employed powerful tools for investigating self-assembled systems under equilibrium conditions. There is a growing interest in studying dynamic structural transitions under non-equilibrium conditions, mainly by combining SAXS with a stopped flow apparatus or a light source to gain insight on the structural mechanism and the possible formation of intermediate phases (Pawate et al., 2015; Angelov et al., 2015; Narayanan, 2009; Yaghmur, Laggner, Sartori \& Rappolt, 2008). However, stopped flow apparatuses are rather complex, and excessive amounts of sample are typically wasted (Bleul et al., 2011). In this context, combining microfluidics with X-ray techniques allows the characterization of self-assembled structures after a very specific reaction time down to a few microseconds, only limited by the resolution of the X-ray beam. Thus, a shorter detection time can be obtained by using smaller X-ray beam diameters. Microfluidics provides a better control over the experimental parameters and therefore allows maintaining reproducible experimental conditions between samples, avoiding many of the typical sources of variation in experimental data. Furthermore, sample conditions such as concentrations and mixing times can easily be changed 'on the fly' when using microfluidic devices as opposed to pre-loaded titer plates. Finally, better time management of experiments at a synchrotron beamline can be achieved, which is an important issue owing to the high operating costs of these facilities and the limited available time. In a pioneering study combining microfluidics with X-ray techniques using hydrodynamic focusing, Pollack et al. (1999) demonstrated the compactness of cytochrome c. Other groups have since worked on similar setups for investigating protein folding (Brennich et al., 2011; Saldanha et al., 2016; Lafleur et al., 2011; Graceffa et al., 2013; Akiyama et al., 2002; Uzawa et al., 2004; Panine et al., 2006).

In a recent report by Weinhausen \& Köster (2013), a new type of X-ray-compatible microfluidic device, based on a UV- curable adhesive and thin Kapton films and featuring silicon nitride membrane windows for X-ray measurements on whole biological cells, was presented. The fabrication method used to prepare the microfluidic system requires the use of photolithography (masks), which can be more expensive and cumbersome compared to fabrication using in-house precision micromilling. A microfluidic chip made from a micromilled $1.7 \mathrm{~mm}$-thick polystyrene substrate featuring thermally bonded $125 \mu \mathrm{m}$-thick polystyrene X-ray-compatible windows has been reported (Lafleur et al., 2011). However, micromilling of individual chips followed by thermal bonding is both time consuming and labor intensive compared to molding techniques. In another approach (Otten et al., 2005; Dootz et al., 2006; Pfohl et al., 2007), stainless steel and Kapton were used as the main materials to prepare microfluidic systems. Similar challenges were presented in other studies (Akiyama et al., 2002; Uzawa et al., 2004).

Recently, photocurable adhesives based on thiol-ene (TE) and off-stoichiometry thiol-ene (OSTE) chemistry have attracted much attention as an alternative material for chip production (Carlborg et al., 2011; Ashley et al., 2011; Baylor et al., 2011; Lafleur et al., 2013, 2015). TE/OSTE-based polymers allow for the easy bench top fabrication of microfluidic chips featuring SAXS-compatible polystyrene (PS) windows. PS has previously been demonstrated to be a suitable material with regards to transparency and scattering background in SAXS analyses (Toft et al., 2008). In this work, we present a study on the use of OSTE for the fabrication of microfluidic chips optimal for X-ray studies. As proof of concept, the thiol-ene chips were used and combined with synchrotron SAXS for investigating real-time calcium-triggered dynamic structural alterations in the self-assembled interiors of cubosome nanoparticles based on binary phytantriol/dioleoylphosphatidylglycerol (PHYT/DOPG) mixtures. Calcium ions play an important role in different biological processes, including protein regulation and transport of molecules across biological membranes, owing to the strong interactions of calcium ions with anionic phospholipids embedded in biological membranes (Yaghmur, Laggner, Sartori \& Rappolt, 2008; Faraudo \& Travesset, 2007; Hong et al., 1982; Papahadjopoulos $\&$ Poste, 1975). However, evaluation of the effect of $\mathrm{Ca}^{2+}$ ions on real biological membranes and multicomponent lipid systems is difficult because of their complexity. In this context, there is a growing interest in understanding the effect of $\mathrm{Ca}^{2+}$ ions and other divalent ions on negatively charged liposomes as model simple membranes and also on other related nanostructured aqueous dispersions including cubosomes and hexosomes (Lee et al., 2013; Muir et al., 2012; Yaghmur, Sartori \& Rappolt, 2011; Yaghmur, Laggner, Sartori \& Rappolt, 2008). Using the OSTE-based microfluidic device, $\mathrm{Ca}^{2+}$ ions were mixed with negatively charged PHYT/DOPG-based cubosome nanoparticles enveloping a well ordered inverse bicontinuous cubic $\operatorname{Im} 3 m$ phase. The bonded polystyrene windows allowed the collection of SAXS data in a time frame between 0.5 and $5.5 \mathrm{~s}$ from the initial mixing time, where PIPES buffer containing $\mathrm{Ca}^{2+}$ ions was mixed with the PHYT/ DOPG solution at the channel junction. 


\section{Experimental section}

\subsection{Materials for preparing cubosomes}

Phytantriol (PHYT: 3,7,11,15,-tetramethyl-1,2,3-hexadecanetriol) with a purity of $>96.4 \%$ (from product specifications) was purchased from DSM Nutritional Products Ltd (Basel, Switzerland). DOPG (1,2-dioleoyl-sn-glycero-3phospho-rac-glycerol; purity 99.2\%) was purchased from LIPOID GmbH (Ludwigshafen, Germany). Pluronic F127 was a gift from BASF SE (Ludwigshafen, Germany). PIPES buffer of $10 \mathrm{~m} M$ was made using piperazine-1,4-bis(2-ethanesulfonic acid) purchased from Merck KGaA (Darmstadt, Germany), which was titrated with $\mathrm{NaOH}$ to reach a $\mathrm{pH}$ of 7.00. PIPES buffer containing $272 \mathrm{mMC \textrm {Ca } ^ { 2 + }}$ was made by dissolving an appropriate amount of $\mathrm{CaCl}_{2}$ salt in the $10 \mathrm{mM}$ PIPES buffer. Ethanol of $98 \%$ purity from Kemetyl A/S (Køge, Denmark) was used for cleaning purposes. The molecular structures of lipids used in the present work are shown in Fig. 1.

\subsection{Materials for producing the microfluidic chip and chip holder}

Pentaerythritoltetrakis (3-mercaptopropionate, 'tetrathiol') and triallyl-1,3,5-triazine-2,4,6(1H,3H,5H)-trione ('triallyl') were obtained from Sigma Aldrich (Brøndby, DK). A Sylgard 184 poly(dimethylsiloxane) (PDMS) elastomer kit was obtained from Dow Corning (Midland, MI, USA). Polycarbonate and poly(methyl methacrylate) (PMMA) sheets of $5 \mathrm{~mm}$ in thickness $(10 \times 10 \mathrm{~cm}$ in width and length $)$ were purchased from Nordisk Plast A/S (Randers, DK). A Lee Minstac 062 connectors fitting assembly and tool kit were obtained from Lee Company Scandinavia AB (Vällingby, Sweden). Polystyrene films of $125 \mu \mathrm{m}$ thickness were purchased from Goodfellow Cambridge Ltd (Huntingdon, UK).

\subsection{Microfluidic device fabrication}

The chips were designed using computer-aided-design software (Autodesk Inventor Professional 2014, San Rafael, CA, USA). The microfluidic system as presented in Fig. 2 was prepared by sandwiching a mixture of tetrathiol and triallyl monomers prepared at different weight ratios between two thin polystyrene films. Tetrathiol and triallyl were mixed at a ratio of 60:40, respectively. To produce the thiol-ene chips, a master mold was fabricated by micromilling of PMMA substrates. The PMMA master mold is used to fabricate new PDMS molds by replica molding. The PDMS acts as a mold for the photocuring of the OSTE chips and its flexibility allowed for easy demolding of the cured OSTE and reuse. The PMMA mold was manufactured by high-precision micromilling (Minitech 3, Minitech Machinery Corporation, Norcross, GA, USA), allowing the creation of features down to $100 \mu \mathrm{m}$. The PDMS mixture was prepared by mixing the PDMS elastomer with curing agent at a ratio of 1:10, then stirred for a few minutes to ensure complete mixing. The PDMS mold was made by pouring the PDMS mixture into the PMMA mold and curing at $333 \mathrm{~K}$ for $2 \mathrm{~h}$. Prior to filling of the PDMS mold, the OSTE monomers were mixed and $1 \%$ photo-initiator (Lucirin TPO-L, BASF, Switzerland) was added. When the PDMS mold was filled with OSTE, the pattern in the mold produced a $225 \mu \mathrm{m}$-thick OSTE layer with a network of $320 \mu \mathrm{m}$-wide channels. These channels of $320 \mu \mathrm{m}$ width correspond to the area through which the X-ray beam travels, and owing to limitations on the dimensions of the X-ray beam $(200 \times 250 \mu \mathrm{m})$ the channels of the chip must remain wider than the beam dimensions. After filling the OSTE mixture into the PDMS mold, a polystyrene sheet was placed on top of the monomer mixture before the first curing step took place. The chip was semi-cured under UV flood illumination for $10 \mathrm{~s}$ on one side (Dymax 5000-EC Series UV curing flood lamp, Dymax Corporation, Torrington, CT, USA, $160 \mathrm{~mW} \mathrm{~cm}^{-2}$ at $365 \mathrm{~nm}$ ). Then, the semi-cured thiol-ene chip was peeled off the PDMS mold. A polystyrene film was placed on the other side of the chip, and the chip was then fully cured for another $10 \mathrm{~s}$ before it was ready for use (Fig. 2). The thiol-ene chip was placed in a sample holder made of $5 \mathrm{~mm}$-thick PMMA with Lee Minstac 062 connectors fitted on the inlets and outlet. Orings were also placed between the thiol-ene chip and the chip holder to ensure sealing.

When designing thiol-ene microfluidic chips for SAXS, it is crucial that nothing adheres to the walls of the channels (or any window that the X-ray radiation has to travel through) since any adhesion to the channel walls significantly affects the detected signal; therefore, a protective coating was applied. In order to coat the walls of the thiol-ene channels, two copolymers based on 3-trimethylsilanylprop-2-yn methacrylate (PMA) and N,N-dimethylacrylamide (DMA) were used. These copolymers were synthesized and provided by the group of Marcella Chiari at ICRM (Milano, Italy). PMA covalently binds to the thiol groups present on the channel surface and thus provides a strong anchoring of the copolymer. DMA is well known to reduce analyte adsorption by hydrophilizing the hydrophobic silica surfaces (Zilio et al., 2015; Cretich et al., 2005; Chiari et al., 2000). The entire process is fast and easy to implement, with UV illumination done within a few minutes. The thiol-ene channels were filled with the copolymer solution using a syringe pump by carefully

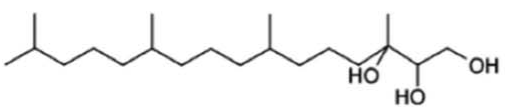

PHYT

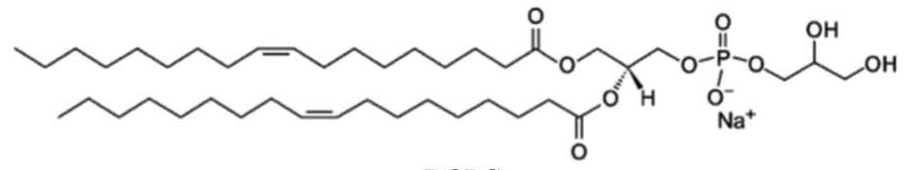

DOPG

Figure 1

Molecular structures of lipids used in the study. 
placing the transfer tube from the syringe on the inlets of the chip. After the channels had been filled with the coating polymer, the chips were exposed to UV light and then flushed with milli-Q water.

Both coated and uncoated chips were tested at the synchrotron SAXS beamline to gain further insight on possible adherence of lipids on the surface of the chips. The lipids remained adhered to the walls after injection of lipid nanoparticles using the uncoated chip (Fig. 3b), whereas there was no indication of the presence of an adhered lipidic layer when performing SAXS experiments using the coated chips (Fig. 3c). It was therefore important to perform the presented experiments with coated chips to guarantee a successful SAXS data analysis.

\subsection{Preparation of DOPG/PHYT dispersions}

DOPG and the polymeric stabilizer Pluronic F127 were dissolved in $10 \mathrm{~m} M$ PIPES buffer with vortex mixing and heating to $323 \mathrm{~K}$. Phytantriol was weighed out into the bottom of a vial and the pre-mixed solution of DOPG with F127 was gently pipetted on top. The concentration of F127 was kept at $1 \%(w / w)$ for two dispersions prepared with PHYT:DOPG at weight ratios of 7:1 and 10:1 and a total lipid content of $6 \%(w /$ $w$ ). An ultrasonic processor (Sonics Vibra Cell VCX 130, power $130 \mathrm{~W}$, frequency $20 \mathrm{kHz}$ ) was used for $10 \mathrm{~min}$ in pulse mode (1 s pulse interrupted by $2 \mathrm{~s}$ break) at $20 \%$ of maximum amplitude until well dispersed milky solutions were obtained.

\subsection{Synchrotron SAXS setup}

SAXS measurements were performed at the beamline I911SAXS at the MAX-lab synchrotron facility (MAX II storage ring, Lund University, Sweden) at an operating electron energy of $1.5 \mathrm{GeV}$ and a wavelength of $0.91 \AA$ (Labrador et al., 2013). The dimensions of the X-ray beam were set to a width of $250 \mu \mathrm{m}$ and a height of $200 \mu \mathrm{m}$. Attempts were made to focus the X-ray beam to smaller dimensions; however, the
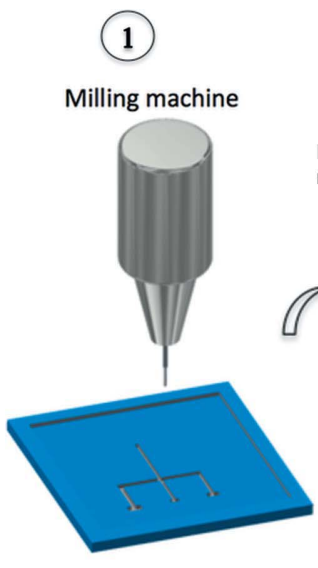

PMMA plate

PDMS

Thiol-ene

(1)
(2)

Pour liquid PDMS into milled PMMA mold then cure PDMS at $333 \mathrm{~K}$ for $2 \mathrm{~h}$.

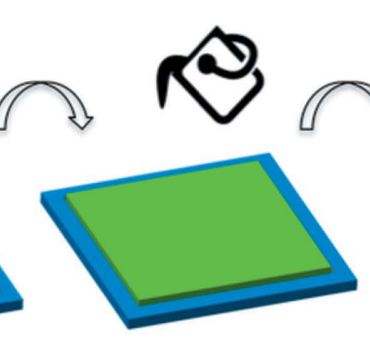

(3)

Pour liquid thiol-ene into PDMS mold then place polystyrene film then cure thiol-ene by exposing to UV for $10 \mathrm{~s}$.

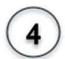

Turn thiol-ene chip then place the other polystyrene film then cure again under UV light for $10 \mathrm{~s}$
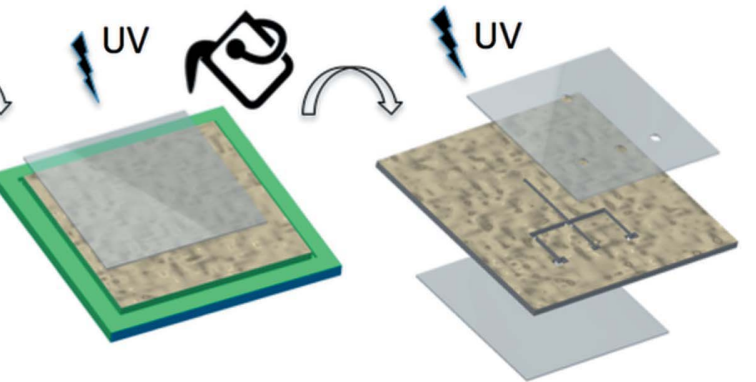

Polystyrene films(125 $\mu \mathrm{m}$ thick)

(a)

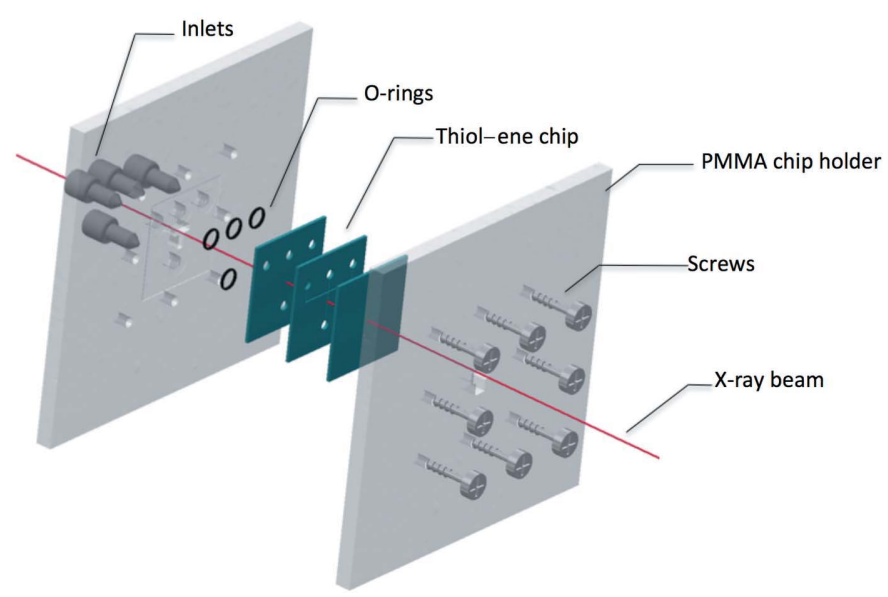

(b)

Figure 2

(a) Fabrication method for preparing thiol-ene chips. (b) Exploded view of the microfluidic setup. 


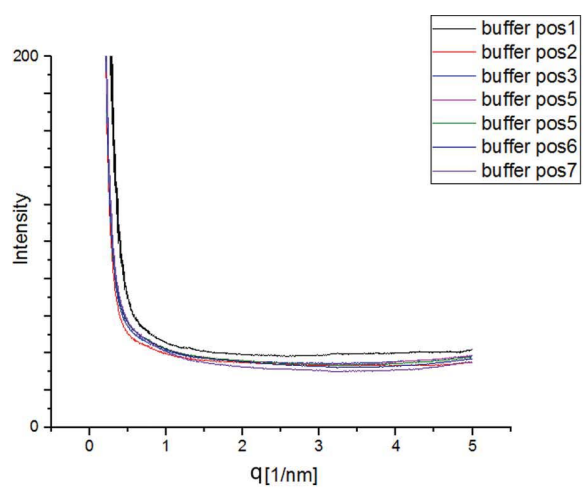

(a)

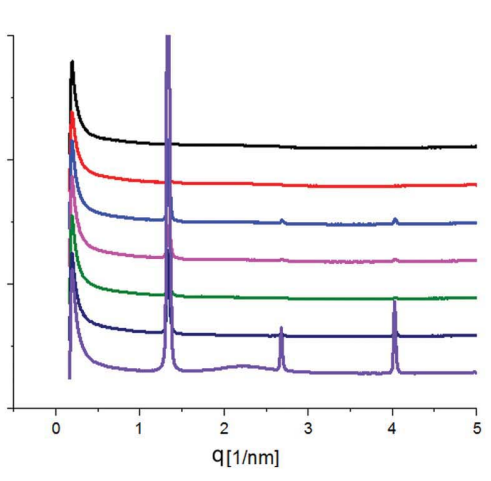

(b)

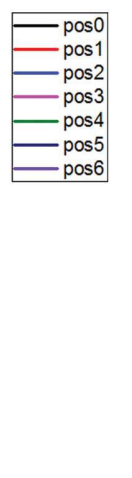

Figure 3

SAXS measurements performed on a microfluidic chip to test the adherence of lipid residue onto the channel walls of the chip. Measurements were performed starting from the intersection area between the side channels and the centre channel and moving downward towards the exit of the main channel. The figure shows the SAXS signal from $(a)$ an uncoated thiol-ene chip with only buffer flowing through the channels (before injecting lipidic formulations), $(b)$ the uncoated thiol-ene chip after injection of a lipidic formulation for testing and flushing of the microfluidic chip with buffer, and (c) a coated chip after injection of lipidic formulations and flushing with buffer. It is evident that after injection of the lipidic formulations in the uncoated chip $(b)$, and after SAXS measurements had been performed, the lipids remained adhered on the walls of the chip, unlike in (c), where no lipids remained adhered to the walls.

intensity of the X-ray beam decreased significantly and therefore accurate SAXS data analysis was not permitted. The chip was aligned in the X-ray beam in a way that the width of the X-ray beam is perpendicular to the direction of the flow. The measurements were performed under vacuum to minimize background scattering. The detector covered a $q$ range $(q=4 \pi \sin \theta / \lambda)$ of $0.01-0.40 \AA^{-1}$, where $q$ is the momentum transfer, $\lambda$ is the wavelength and $2 \theta$ is the scattering angle. Silver behenate $\left[\mathrm{CH}_{3}-\left(\mathrm{CH}_{2}\right)_{20}-\mathrm{COOAg}\right]$ with a $d$ spacing value of $58.4 \AA$ was used as a standard to calibrate the scattering momentum. The scattering patterns were recorded with a two-dimensional photon counting pixel detector (Pilatus $1 \mathrm{M}$, Dectris Ltd, Baden, Switzerland) using collection times of $60 \mathrm{~s}$. All measurements were carried out at room temperature. Different total volumetric flow rates ranging from 4 to $16 \mu \mathrm{min}^{-1}$ were used. The X-ray beam was scanned along the microfluidic channel at seven different positions down the centre channel with a spacing of $1 \mathrm{~mm}$ between each position (Fig. 4). SAXS measurement was performed before mixing with calcium ions at an appropriate position above the channel

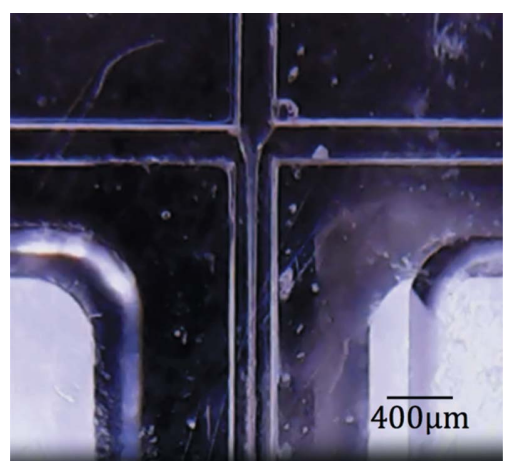

Figure 4

Micrograph of the microfluidic chip, showing hydrodynamic focusing of the PIPES buffer containing $\mathrm{Ca}^{2+}$ ions as sheath fluid (side channels) and PHYT/DOPG-based dispersion in the centre stream. intersection (position 1) in order to characterize the structure of the dispersion before mixing with $\mathrm{Ca}^{2+}$ ions. This can be clearly seen in Fig. 5(a) (first SAXS pattern from top).

\subsection{Dynamic SAXS study of $\mathrm{Ca}^{2+}$ ion-induced structural transitions in PHYT/DOPG dispersions}

Pre-prepared dispersions of binary PHYT/DOPG mixture in $10 \mathrm{~m} M$ PIPES buffer ( $\mathrm{pH} 7.00$ ) were loaded into a $1 \mathrm{ml} \mathrm{B}$. Braun Inject-F Solo syringe. PIPES buffer ( $\mathrm{pH} 7.00)$ with $272 \mathrm{mM} \mathrm{Ca}{ }^{2+}$ ion concentration was loaded into two BD $3 \mathrm{ml}$ syringes. These syringes were connected to the microfluidic device by tubes, and hydrodynamic focusing was achieved by pumping the lipid-containing dispersion through the middle channel and $\mathrm{Ca}^{2+}$ ion-containing PIPES buffer through the two side channels. The flow rate ratio of PHYT/DOPG dispersion and buffer was 1:1 so that the final concentration of $\mathrm{Ca}^{2+}$ was $136 \mathrm{~m} M$ after mixing. The flow rates were in the range of 4-16 $\mu \mathrm{l} \mathrm{min}^{-1}$, which corresponds to linear velocities of between 0.92 and $3.7 \mathrm{~mm} \mathrm{~s}^{-1}$, respectively. The exposure time permitting accurate data analysis with a good signal-tonoise ratio was $60 \mathrm{~s}$ for each position. The width of the focused centre stream is directly related to the flow rate ratio (FRR) between the lipid stream (centre) and the side buffer stream (sheath). The width was calculated using the following equation:

$$
w=\frac{\text { width of channel }}{1+\mathrm{FRR}},
$$

where $w$ is the width of the focused centre stream, FRR is the flow rate ratio between the centre stream and the side buffer stream (sheath), and the width of the channel is $320 \mu \mathrm{m}$. This means that the width of the focused stream in our investigations was $160 \mu \mathrm{m}$ at an FRR of 1:1 (centre to sheath). To estimate the time and position for achieving a complete mixing, the following equation was used: 


$$
t=x^{2} / 2 D
$$

where $t$ is the time in $\mathrm{s}, x$ is the travelled distance measured in $\mathrm{cm}$ and $D$ is the diffusion coefficient measured in $\mathrm{cm}^{2} \mathrm{~s}^{-1}$. The diffusion coefficient of calcium ions was found to be about

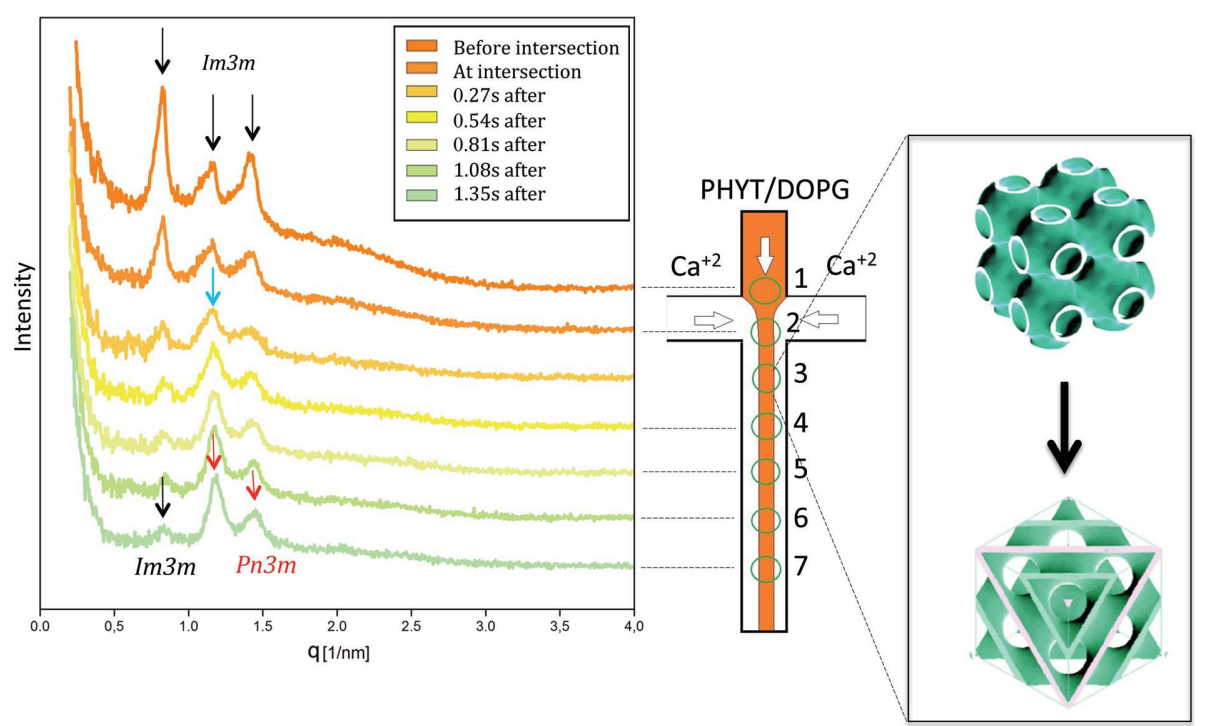

(a)

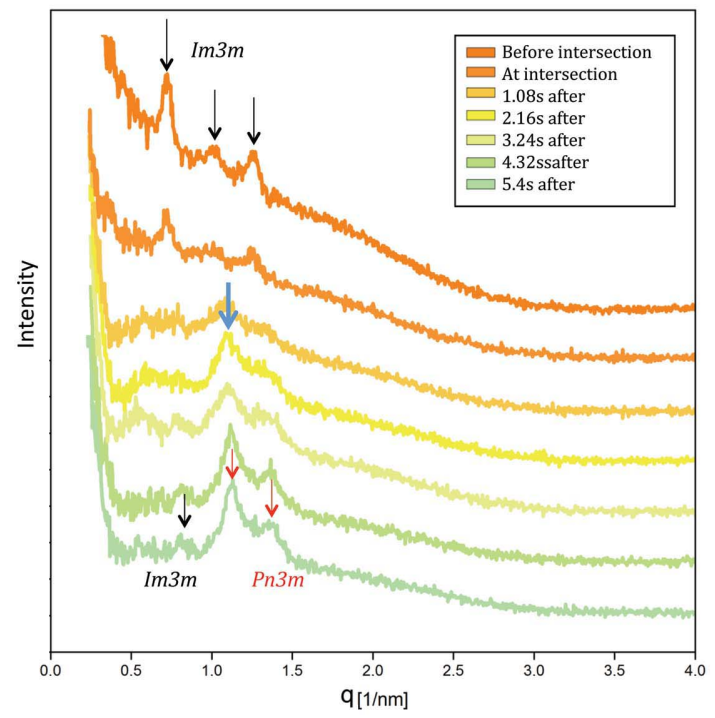

(b)

Figure 5

Time-resolved SAXS experiments displaying the dynamic structural transitions in the interiors of PHYT/DOPG nanoparticles as they were stimulated by an influx of PIPES buffer containing $\mathrm{Ca}^{2+}$ ions. An illustration $(a)$ shows the hydrodynamic focusing chip, with green circles indicating the positions at which SAXS was measured. Position 1 corresponds to the PHYT/DOPG dispersion before mixing, then position 2 is where the PHYT/DOPG dispersion starts mixing with $\mathrm{Ca}^{2+}$ ions, and position 7 is the closest to the outlet of the channel. Each signal on the left corresponds to the relevant position on the microfluidic chip. The weight ratio of PHYT to DOPG was varied from 10:1 to $7: 1$ and the total lipid content was kept constant at $6 \mathrm{wt} \%$. The PHYT/DOPG dispersions were stabilized by $1 \mathrm{wt} \%$ F127. (a) PHYT/DOPG-based dispersion prepared at a PHYT/DOPG weight ratio of 10:1 at a total flow rate of $16 \mu \mathrm{lmin}^{-1}$ which corresponds to a linear velocity of $3.7 \mathrm{~mm} \mathrm{~s}^{-1}$. Inset: schematic illustrations of the internal bicontinuous cubic Pn3m and $\operatorname{Im} 3 m$ phases are adapted from Yaghmur, Sartori \& Rappolt (2006). (b) PHYT/DOPG-based dispersion prepared at a PHYT/ DOPG weight ratio of 7:1 at a total flow rate of $4 \mu 1 \mathrm{~min}^{-1}$, which corresponds to a linear velocity of $0.92 \mathrm{~mm} \mathrm{~s}^{-1}$. The travel time of the fluid from the intersection to the point of SAXS measurement is indicated with corresponding colours. All measurements were performed at room temperature.
$0.79 \times 10^{-5} \mathrm{~cm}^{2} \mathrm{~s}^{-1}$ in a previous report (Samson \& Marchand, 2003). Therefore, it takes an estimated time of $4 \mathrm{~s}$ to to the middle of it).

With the linear velocity of $0.92 \mathrm{~mm} \mathrm{~s}^{-1}$ used in this work, complete mixing could be achieved at approximately $3.68 \mathrm{~mm}$ down the channel, whereas an increase in the linear velocity to $3.7 \mathrm{~mm} \mathrm{~s}^{-1}$ (achieved by varying the volumetric flow rate from 4 to $16 \mu 1 \mathrm{~min}^{-1}$ ) would result in complete mixing at approximately $14.8 \mathrm{~mm}$ down the channel.

It is crucial to find a balance between maintaining a certain concentration of lipid (or lipid mixture) in the centre stream, which permits an accurate and successful SAXS data analysis with good signal-to-noise ratio, and keeping FFR at a reasonable value for achieving a fast complete mixing.

\section{Results}

3.1. Thiol-ene chip and microfluidic setup

The total volumetric flow rates used were in the range between 4 and $16 \mu 1 \mathrm{~min}^{-1}$ which, given the dimensions of the channel, corresponds to a linear velocity of 0.92 and $3.70 \mathrm{~mm} \mathrm{~s}^{-1}$, respectively. The chip fabrication method we developed allowed the rapid prototyping of SAXS-compatible chips within less then 5 min. The chip proved to be very compatible with $\mathrm{X}$-rays as the polystyrene windows exhibited negligible scattering signal (Fig. 3). A camera was mounted on the side of the X-ray beam (parallel to the $\mathrm{X}$-ray beam) and was used to make sure that a steady hydrodynamic focusing was achieved before SAXS measurements were performed. A total of $30-50 \mu \mathrm{l}$ of lipid solution was sufficient for the entire experiment.

\subsection{Structural characterization of PHYT/DOPG dispersions}

PHYT/DOPG dispersions in $10 \mathrm{~m} M$ PIPES buffer ( $\mathrm{pH} 7.00)$ with a total lipid content of $6 \mathrm{wt} \%$ prepared with a PHYT/DOPG weight ratio of 10:1 and $7: 1$ and stabilized with $1 \mathrm{wt} \%$ F127 were investigated. Initially, before mixing with $\mathrm{Ca}^{2+}$ ions, the SAXS 
pattern of the PHYT/DOPG-based dispersion prepared at a PHYT/DOPG weight ratio of 10:1 displayed three peaks with $q$ values of $0.817,1.150$ and $1.405 \mathrm{~nm}^{-1}$ [marked with three black arrows in Fig. 5(a)]. These peaks were identified as the first three reflections of an internal inverse bicontinuous cubic phase of symmetry $\operatorname{Im} 3 m$ with a consistent lattice parameter, $a$, of $10.92 \mathrm{~nm}$ (cubosomes).

It should be noted that PHYT, when dispersed in excess water in the presence of the polymeric stabilizer F127, forms cubosome nanoparticles with an internal inverse bicontinuous cubic $P n 3 m$ phase with a lattice parameter of $6.79 \mathrm{~nm}$. Thus, the obtained result indicates a structural transition from an inverted-type cubic Pn $3 m$ phase to inverted-type cubic $\operatorname{Im} 3 m$ upon the partial replacement of PHYT by a binary lipid mixture of PHYT/DOPG, which is in good agreement with previous studies on the effect of lipid composition on the internal nanostructures of cubosomes and hexosomes based on monoglycerides or PHYT (Azmi, Moghimi \& Yaghmur, 2015; Yaghmur, Sartori \& Rappolt, 2011; Yaghmur, Laggner, Sartori \& Rappolt, 2008; Wadsten-Hindrichsen et al., 2007; Engblom et al., 2001; Engström \& Engström, 1992; Norling et al., 1992).

For the dispersion containing a higher DOPG concentration (PHYT/DOPG weight ratio of 7:1), a cubic $\operatorname{Im} 3 m$ phase with a lattice parameter of $12.43 \mathrm{~nm}$ was detected. Thus, an increase in the lattice parameter of approximately $14 \%$ was achieved on increasing the PHYT/DOPG weight ratio from 10:1 to 7:1 (Table 1). The incorporation of DOPG into the PHYT-water interface has a drastic impact on the self-assembled nanostructure and induces in a concentration-dependent manner an enlargement of the internal hydrophilic nanochannels of the inverted-type cubic Pn $3 m$ phase. As a consequence, structural transitions to an inverted-type cubic $\operatorname{Im} 3 \mathrm{~m}$ phase and even to flattened bilayers [formation of a lamellar $\left(\mathrm{L}_{\alpha}\right)$ phase] can be achieved with a further increase in DOPG content. It was reported that increasing the DOPG concentration induces a transition from a cubic Pn $3 m$ phase via a cubic $\operatorname{Im} 3 m$ phase to a fluid lamellar $\left(\mathrm{L}_{\alpha}\right)$ phase in aqueous dispersions based on unsaturated monoglyceride monoolein (MO)/DOPG mixtures (Yaghmur, Larsen et al., 2011; Yaghmur, Sartori \& Rappolt, 2011).

The critical packing parameter (CPP) is a useful parameter for understanding the role of DOPG in modulating the internal self-assembled nanostructure by taking into consideration the molecular structures of PHYT and DOPG. The CPP is defined as $v_{\mathrm{s}} / a_{0} I$, where $v_{\mathrm{s}}$ is the hydrophobic chain volume, $a_{0}$ is the headgroup area and $I$ is the hydrophobic chain length (Israelachvili et al., 1976). The electrostatic repulsion forces between the negatively charged DOPG molecules incorporated into the electrically neutral PHYTwater interface increase the hydrophilic headgroup area and increase the amount of solubilized water. This exerts stress on the curved bilayers (the cubic $P n 3 m$ phase), favouring a transition to flattened bilayers at a high DOPG concentration. A significant reduction in CPP due to an increase in the hydrophilic headgroup area explains, therefore, the transition to an $\mathrm{L}_{\alpha}$ phase with increasing DOPG content (Yaghmur,
Table 1

Structural lattice parameters of two bicontinuous cubic phases of symmetries $\operatorname{Im} 3 m$ and $P n 3 m$ that were detected in PHYT/DOPG dispersions before and/or after exposure to a buffer containing $272 \mathrm{mM}$ $\mathrm{Ca}^{2+}$ ions.

The total lipid content was kept constant at $6 \mathrm{wt} \%$. The used buffer is $10 \mathrm{~m} M$ PIPES. All dispersions were stabilized by F127 at a concentration of $1 \mathrm{wt} \%$.

PHYT:DOPG ratio of 10:1 (total flow rate of $16 \mu \mathrm{min}^{-1}$ ).

\begin{tabular}{lllll}
\hline Position & Mixing time $(\mathrm{s})$ & $a_{I m 3 m}(\AA)$ & $a_{P n 3 m}(\AA)$ & Bonnet ratio $\dagger$ \\
\hline 1 & Before intersection & 109.2 & - & - \\
2 & At intersection & 109.1 & - & - \\
3 & 0.27 & 107.9 & 77.2 & 1.39 \\
4 & 0.54 & 105.9 & 76.4 & 1.38 \\
5 & 0.81 & 105.8 & 76.0 & 1.39 \\
6 & 1.08 & 105.0 & 75.8 & 1.38 \\
7 & 1.35 & 106.7 & 75.4 & 1.41 \\
\hline
\end{tabular}

PHYT:DOPG ratio of 7:1 (total flow rate of $4 \mu 1 \mathrm{~min}^{-1}$ ).

\begin{tabular}{lllll}
\hline Position & Mixing time $(\mathrm{s})$ & $a_{\operatorname{Im} 3 m}(\AA)$ & $a_{P n 3 m}(\AA)$ & Bonnet ratio $\dagger$ \\
\hline 1 & $\begin{array}{l}\text { Before intersection } \\
\text { At intersection }\end{array}$ & $\begin{array}{l}124.3 \\
\text { Unidentified } \\
\text { structure } \ddagger\end{array}$ & - & - \\
3 & 1.08 & $\begin{array}{r}\text { Unidentified } \\
\text { structure } \ddagger\end{array}$ & - & - \\
& & Unidentified & 81.2 & - \\
4 & 2.16 & structure $\ddagger$ & & - \\
5 & 3.24 & 111.7 & 82.0 & 1.36 \\
6 & 4.32 & 108.8 & 79.9 & 1.36 \\
7 & 5.4 & 109.8 & 79.4 & 1.38 \\
\hline
\end{tabular}

$\dagger$ The Bonnet ratio of the two phases is shown where applicable. $\ddagger$ The signal-to-noise ratio was very low and not sufficient to ensure successful SAXS analysis.

Sartori \& Rappolt, 2011). Briefly, DOPG tends to form the fluid lamellar $\left(\mathrm{L}_{\alpha}\right)$ phase in excess water at ambient temperatures, whereas PHYT tends to form nonlamellar phases. Therefore, the self-assembled nanostructure in these dispersions depends on the PHYT/DOPG molar ratio. In other words, the internal nanostructure is sensitive to alterations in the lipid composition.

\section{3. $\mathrm{Ca}^{2+}$ ion-induced dynamic structural transitions in PHYT/DOPG-based cubosomes}

Two experiments were performed with dispersions prepared with binary PHYT/DOPG mixtures prepared at PHYT/DOPG weight ratios of 10:1 and 7:1 to explore the effect of mixing the negatively charged cubosomes with PIPES buffer containing calcium ions. As presented in Fig. 5, it was possible to monitor alterations in the internal nanostructures of cubosomes within a few seconds as they approached the channel intersection and started mixing with the $\mathrm{Ca}^{2+}$ ioncontaining PIPES buffer ( $\mathrm{pH}$ 7.0). Note that the positions where the X-ray beam hit the chip were the same; however, the reaction time that corresponds to each position differs, depending on a corresponding change in the applied flow rate (Fig. 5). This means that the same chip was used for both dispersions (PHYT/DOPG 10:1 and 7:1) and the SAXS patterns were recorded at identical positions, but with different flow rates and hence different reaction times. 
For the PHYT/DOPG weight ratio of $10: 1$, as shown in Fig. 5(a), the exposure of the negatively charged PHYT/ DOPG cubosome nanoparticles with internal inverse bicontinuous cubic $\operatorname{Im} 3 m$ phase to $\mathrm{Ca}^{2+}$ ions induced a significant effect on the structure. There is an indication of the occurrence of a new phase shortly after exposure of these nanoparticles to $\mathrm{Ca}^{2+}$ ions. The first reflection of this new coexisting phase started to appear at a reaction time of $0.27 \mathrm{~s}$ with a $q$ value of about $1.15 \mathrm{~nm}^{-1}$ [marked with a blue arrow in Fig. 5(a)] and overlapped with the second reflection of the bicontinuous cubic $\operatorname{Im} 3 m$ phase. The assignment of this phase is based on the following: (1) an increase in the intensity of the second reflection of the cubic $\operatorname{Im} 3 m$ phase as compared with its first strongest reflection appearing at a $q$ value of about $0.82 \mathrm{~nm}^{-1}$, indicating the occurrence of a new phase; (2) the development of this coexisting new phase as moving down the intersection (reaction times longer than $0.27 \mathrm{~s}$; Fig. 5a); (3) previous studies on the effect of $\mathrm{Ca}^{2+}$ ions on MO/DOPG dispersions by combining SAXS with a stopped flow apparatus (Yaghmur, Sartori \& Rappolt, 2011). This newly formed coexisting phase was identified as an inverse cubic phase of symmetry Pn3m and its first two reflections were best distinguished at $1.35 \mathrm{~s}$ after mixing [marked with red arrows in Fig. 5(a)]. They were detected at $q$ values of 1.185 and $1.435 \mathrm{~nm}^{-1}$. A slight decrease in the calculated lattice parameter was found as this phase develops over time when moving down the intersection (Table 1). As the newly formed cubic $P n 3 m$ phase started to appear, the intensity of the first and strongest reflection of the cubic $\operatorname{Im} 3 m$ phase was significantly reduced with increasing mixing time. Traces of this phase coexisting with a more developed cubic $P n 3 m$ phase were still recognizable on the SAXS spectra after $1.35 \mathrm{~s}$ of mixing with $\mathrm{Ca}^{2+}$ ions.

Another experiment was performed on the effect of $\mathrm{Ca}^{2+}$ ions on a dispersion containing a higher DOPG concentration (PHYT/DOPG weight ratio of 7:1). It was evident that $\mathrm{Ca}^{2+}$ ions induce a structural transformation from an internal inverse cubic $\operatorname{Im} 3 \mathrm{~m}$ phase with a lattice parameter of $12.43 \mathrm{~nm}$ to a biphasic nanostructure consisting of a cubic $\operatorname{Im} 3 \mathrm{~m}$ phase with a lattice parameter of $10.98 \mathrm{~nm}$ and a cubic Pn3m phase with a lattice parameter of $7.94 \mathrm{~nm}$ (Fig. $5 b$ ). It took a much longer time for the cubic Pn3m phase to develop in this formulation. A sharp peak signifying the cubic Pn $3 m$ phase only became apparent [marked with a blue arrow in Fig. 5(b)] after $2.2 \mathrm{~s}$ of mixing. This phase became distinguishable and its lattice parameter started to slightly decrease from 8.12 to $7.94 \mathrm{~nm}$ (a decrease of 2\%) after $3.16 \mathrm{~s}$. The cubic Im $3 m$ phase, however, started to vanish much faster and only traces of this phase were found with increasing mixing time. This phase also suffered a large decrease in its lattice parameter from 12.43 to $10.98 \mathrm{~nm}$ after $5.4 \mathrm{~s}$ of hydrodynamic mixing. The coexistence of the bicontinuous cubic Pn3m and Im $3 m$ phases is governed by an interesting relationship described by the Gauss-Bonnet theorem (Hyde, 1996). When a phase transition occurs in excess water, the ratio of the parent and product lattice parameters follows the so-called Bonnet ratio (Hyde, 1996). The reported theoretical Bonnet ratio for a biphasic $\operatorname{Im} 3 \mathrm{~m} /$
Pn3m structure is 1.27 (Hyde, 1996; Larsson \& Tiberg, 2005). In the present work, the Bonnet ratio for these two detected phases ranged between 1.36 and 1.41 (Table 1). Note that previous studies on the structural characteristics of different lipid dispersions reported Bonnet ratios for coexisting $\mathrm{Im} 3 \mathrm{~m} /$ $P n 3 m$ phases in the range of 1.31-1.36 (Larsson \& Tiberg, 2005; Yaghmur, Laggner, Almgren \& Rappolt, 2008; Guillot et al., 2010; Nilsson et al., 2014). Thus, the obtained values are in reasonable agreement with the previous reports. The structural changes on exposure of $\mathrm{Ca}^{2+}$ ions to cubosomes are attributed to electrostatic interactions between the calcium ions and the negatively charged headgroups of the DOPG molecules. It was reported that different negatively charged phospholipids form strong complexes with $\mathrm{Ca}^{2+}$ ions by lessening electrostatic repulsion forces at the lipid-water interface, which results in a release of water molecules (dehydration of the hydrophilic headgroups) (Yaghmur, Sartori \& Rappolt, 2011) and a tighter packing of the fatty chains (the condensing effect) (Pedersen et al., 2006). It has been further reported that the concentration dependence of the condensing effect for $\mathrm{Ca}^{2+}$ induces a decrease in the water permeability of DOPG and its lateral diffusion constant $\left(D_{\mathrm{L}}\right)$ (Filippov et al., 2009). In addition to electrostatic interactions, the entropy gain due to the release of water molecules from the hydration shells of the hydrophilic headgroups of negatively charged phospholipids (the dehydration effect) has been found to play an important role in modulating the selfassembled nanostructures (Garidel \& Blume, 1999; Garidel et al., 2000).

It was suggested by Lehrmann \& Seelig (1994) that the mechanism behind the strong binding of $\mathrm{Ca}^{2+}$ ions to negatively charged phospholipids consists of two major steps:

(1) Increasing the local $\mathrm{Ca}^{2+}$ concentration: the divalent ions are attached to the anionic groups of the lipid molecules, resulting in an increase in the local $\mathrm{Ca}^{2+}$ concentration at the lipid-water interface.

(2) Interactions of $\mathrm{Ca}^{2+}$ ions with the phosphate group: coordination complexes form as a result of the direct interactions of $\mathrm{Ca}^{2+}$ ions with the phosphate group of one or more lipid molecules (Lehrmann \& Seelig, 1994; Yaghmur, Laggner, Sartori \& Rappolt, 2008).

\section{Conclusion}

A simple microfabrication technique for producing microfluidic chips compatible with synchrotron X-ray techniques is presented in this work. The designed microfluidic system is based on UV-curable thiol-ene-chemistry-based polymers, with X-ray-compatible polystyrene windows. It is simple, low cost, easy and rapid to produce, disposable, and reduces sample consumption to few tens of microlitres. Moreover, the microfluidic system allows the rapid changing of samples and could be useful for a variety of investigations such as protein folding. As proof of concept, to demonstrate the functionality and suitability of the microfluidic device for X-ray experiments, the effect of calcium ions on negatively charged cubosomes was investigated. 
A successful combination of synchrotron SAXS with the microfluidic device was used to monitor in real time calciumtriggered structural changes on direct exposure of negatively charged cubosomes to buffer containing $\mathrm{Ca}^{2+}$ ions. It was possible to follow the internal structural transitions of PHYT/ DOPG-based cubosomes from cubic $\operatorname{Im} 3 m$ to $P n 3 m$ phases in a time range of $0.5-5.5 \mathrm{~s}$. These results demonstrate the efficiency of the microfluidic system and its usefulness for obtaining information on dynamic structural transitions in cubosomes and other related nanostructured aqueous dispersions used as nanocarriers for delivery of drugs and imaging probes.

\section{Acknowledgements}

The authors would like to thank Camilla Foged and Susan Weng Larsen from the University of Copenhagen for discussions and valuable input. The authors also thank the group of Marcella Chiari at ICRM (Milan, Italy) for providing the necessary polymers for coating our chips. Moreover, the authors thank the staff at the Max IV facility for their support, and the Copenhagen University Neutron and X-ray Techniques (CoNeXT) initiative and DanScatt, which is part of the Danish Agency for Science, Technology and Innovation, for providing funding for travel to experiments.

\section{References}

Akiyama, S., Takahashi, S., Kimura, T., Ishimori, K., Morishima, I., Nishikawa, Y. \& Fujisawa, T. (2002). Proc. Natl Acad. Sci. USA, 99, 1329-1334.

Angelov, B., Angelova, A., Drechsler, M. \& Lesieur, S. (2015). J. Surf. Investig. X-ray Synchrotron Neutron Tech. 9, 105-110.

Anna, S. L., Bontoux, N. \& Stone, H. A. (2003). Appl. Phys. Lett. 82, 364-366.

Ashley, J. F., Cramer, N. B., Davis, R. H. \& Bowman, C. N. (2011). Lab Chip, 11, 2772-2778.

Azmi, I. D., Moghimi, S. M. \& Yaghmur, A. (2015). Ther. Deliv. 6, $1347-1364$

Azmi, I. D., Wibroe, P. P., Lu, L.-P., Kazem, A. I., Amentisch, H., Moghimi, S. M. \& Yaghmur, A. (2016). J. Control. Rel. 239, 1-9.

Azmi, I. D. M., Wu, L., Wibroe, P. P., Nilsson, C., Østergaard, J., Stürup, S., Gammelgaard, B., Urtti, A., Moghimi, S. M. \& Yaghmur, A. (2015). Langmuir, 31, 5042-5049.

Baylor, M.-E., Boyne, R. W., Cramer, N. B., Bowman, C. N. \& McLeod, R. R. (2011). Proc. SPIE, 8097, 80970P.

Bleul, R., Ritzi-Lehnert, M., Höth, J., Scharpfenecker, N., Frese, I., Düchs, D., Brunklaus, S., Hansen-Hagge, T. E., Meyer-Almes, F.-J. \& Drese, K. S. (2011). Anal. Bioanal. Chem. 399, 1117-1125.

Brennich, M. E., Nolting, J.-F., Dammann, C., Nöding, B., Bauch, S., Herrmann, H., Pfohl, T. \& Köster, S. (2011). Lab Chip, 11, 708-716.

Carlborg, C. F., Haraldsson, T., Öberg, K., Malkoch, M. \& van der Wijngaart, W. (2011). Lab Chip, 11, 3136-3147.

Chiari, M., Cretich, M., Damin, F., Ceriotti, L. \& Consonni, R. (2000). Electrophoresis, 21, 909-916.

Cretich, M., Chiari, M., Pirri, G. \& Crippa, A. (2005). Electrophoresis, 26, 1913-1919.

Dehsorkhi, A., Castelletto, V. \& Hamley, I. W. (2014). J. Pept. Sci. 20, 453-467.

Dittrich, P. S. \& Manz, A. (2005). Anal. Bioanal. Chem. 382, 17711782.

Dittrich, P. S. \& Manz, A. (2006). Nat. Rev. Drug Discov. 5, 210-218.

Dootz, R., Otten, A., Köster, S., Struth, B. \& Pfohl, T. (2006). J. Phys. Condens. Matter, 18, S639-S652.
Edel, J. B., Beard, N. P., Hofmann, O., deMello, J. C., Bradley, D. D. C. \& deMello, A. J. (2004). Lab Chip, 4, 136-140.

Engblom, J., Miezis, Y., Nylander, T., Razumas, V. \& Larsson, K. (2001). Surface and Colloid Science, Progress in Colloid and Polymer Science, Vol. 116, edited by V. Razumas, B. Lindman \& T. Nylander, pp. 9-15. Berlin, Heidelberg: Springer.

Engström, S. \& Engström, L. (1992). Int. J. Pharm. 79, 113-122.

Faraudo, J. \& Travesset, A. (2007). Biophys. J. 92, 2806-2818.

Filippov, A., Orädd, G. \& Lindblom, G. (2009). Chem. Phys. Lipids, 159, 81-87.

Gañán-Calvo, A. M. \& Gordillo, J. M. (2001). Phys. Rev. Lett. 87, 274501.

Garidel, P. \& Blume, A. (1999). Langmuir, 15, 5526-5534.

Garidel, P., Förster, G., Richter, W., Kunst, B. H., Rapp, G. \& Blume, A. (2000). Phys. Chem. Chem. Phys. 2, 4537-4544.

Graceffa, R., Nobrega, R. P., Barrea, R. A., Kathuria, S. V., Chakravarthy, S., Bilsel, O. \& Irving, T. C. (2013). J. Synchrotron Rad. 20, 820-825.

Guha, S., Perry, S. L., Pawate, A. S. \& Kenis, P. J. A. (2012). Sens. Actuators B Chem. 174, 1-9.

Guillot, S., Salentinig, S., Chemelli, A., Sagalowicz, L., Leser, M. E. \& Glatter, O. (2010). Langmuir, 26, 6222-6229.

Hansen, C. L., Skordalakes, E., Berger, J. M. \& Quake, S. R. (2002). Proc. Natl Acad. Sci. USA, 99, 16531-16536.

Hong, K., Düzgüneş, N. \& Papahadjopoulos, D. (1982). Biophys. J. 37, 297-305.

Hyde, S. T. (1996). Curr. Opin. Solid State Mater. Sci. 1, 653-662.

Israelachvili, J. N., Mitchell, D. J. \& Ninham, B. W. (1976). J. Chem. Soc. Faraday Trans. 2, 72, 1525-1568.

Labrador, A., Cerenius, Y., Svensson, C., Theodor, K. \& Plivelic, T. (2013). J. Phys. Conf. Ser. 425, 072019.

Lafleur, J. P., Kwapiszewski, R., Jensen, T. G. \& Kutter, J. P. (2013). Analyst, 138, 845-849.

Lafleur, J. P., Senkbeil, S., Novotny, J., Nys, G., Bøgelund, N., Rand, K. D., Foret, F. \& Kutter, J. P. (2015). Lab Chip, 15, 2162-2172.

Lafleur, J. P., Snakenborg, D., Nielsen, S. S., Møller, M., Toft, K. N., Menzel, A., Jacobsen, J. K., Vestergaard, B., Arleth, L. \& Kutter, J. P. (2011). J. Appl. Cryst. 44, 1090-1099.

Larsson, K. \& Tiberg, F. (2005). Curr. Opin. Colloid Interface Sci. 9, $365-369$.

Lee, H.-Y., Hashizaki, K., Diehn, K. \& Raghavan, S. R. (2013). Soft Matter, 9, 200-207.

Lehrmann, R. \& Seelig, J. (1994). Biochim. Biophys. Acta, 1189, 8995.

Link, D. R., Anna, S. L., Weitz, D. A. \& Stone, H. A. (2004). Phys. Rev. Lett. 92, 054503.

Liu, J., White, I. \& DeVoe, D. L. (2011). Anal. Chem. 83, 2119-2124.

Llobera, A., Demming, S., Wilke, R. \& Büttgenbach, S. (2007). Lab Chip, 7, 1560-1566.

Marchand, G., Broyer, P., Lanet, V., Delattre, C., Foucault, F., Menou, L., Calvas, B., Roller, D., Ginot, F., Campagnolo, R. \& Mallard, F. (2008). Biomed. Microdevices, 10, 35-45.

Muir, B. W., Zhen, G., Gunatillake, P. \& Hartley, P. G. (2012). J. Phys. Chem. B, 116, 3551-3556.

Narayanan, T. (2009). Curr. Opin. Colloid Interface Sci. 14, 409-415.

Nie, Z., Seo, M., Xu, S., Lewis, P. C., Mok, M., Kumacheva, E., Whitesides, G. M., Garstecki, P. \& Stone, H. A. (2008). Microfluid. Nanofluid. 5, 585-594.

Nilsson, C., Barrios-Lopez, B., Kallinen, A., Laurinmäki, P., Butcher, S. J., Raki, M., Weisell, J., Bergström, K., Larsen, S. W., Østergaard, J., Larsen, C., Urtti, A., Airaksinen, A. J. \& Yaghmur, A. (2013). Biomaterials, 34, 8491-8503.

Nilsson, C., Østergaard, J., Larsen, S. W., Larsen, C., Urtti, A. \& Yaghmur, A. (2014). Langmuir, 30, 6398-6407.

Norling, T., Lading, P., Engström, S., Larsson, K., Krog, N. \& Nissen, S. S. (1992). J. Clin. Periodontol. 19, 687-692.

Otten, A., Köster, S., Struth, B., Snigirev, A. \& Pfohl, T. (2005). J. Synchrotron Rad. 12, 745-750. 
Panine, P., Finet, S., Weiss, T. M. \& Narayanan, T. (2006). Adv. Colloid Interface Sci. 127, 9-18.

Papahadjopoulos, D. \& Poste, G. (1975). Biophys. J. 15, 945-948.

Pawate, A. S., Šrajer, V., Schieferstein, J., Guha, S., Henning, R., Kosheleva, I., Schmidt, M., Ren, Z., Kenis, P. J. A. \& Perry, S. L. (2015). Acta Cryst. F71, 823-830.

Pedersen, U. R., Leidy, C., Westh, P. \& Peters, G. H. (2006). Biochim. Biophys. Acta, 1758, 573-582.

Pfohl, T., Otten, A., Köster, S., Dootz, R., Struth, B. \& Evans, H. M. (2007). Biomacromolecules, 8, 2167-2172.

Pollack, L., Tate, M. W., Darnton, N. C., Knight, J. B., Gruner, S. M., Eaton, W. A. \& Austin, R. H. (1999). Proc. Natl Acad. Sci. USA, 96, 10115-10117.

Polte, J., Erler, R., Thünemann, A. F., Sokolov, S., Ahner, T. T., Rademann, K., Emmerling, F. \& Kraehnert, R. (2010). ACS Nano, 4, 1076-1082.

Price, A. K. \& Culbertson, C. T. (2007). Anal. Chem. 79, 2614-2621.

Saldanha, O., Brennich, M. E., Burghammer, M., Herrmann, H. \& Köster, S. (2016). Biomicrofluidics, 10, 024108.

Samson, E. \& Marchand, J. (2003). Mater. Struct. 36, 156-165.

Shrestha, L. K., Strzelczyk, K. M., Shrestha, R. G., Ichikawa, K., Aramaki, K., Hill, J. P. \& Ariga, K. (2015). Nanotechnology, 26, 204002.

Sia, S. K. \& Whitesides, G. M. (2003). Electrophoresis, 24, 3563-3576.

Stavis, S. M., Edel, J. B., Samiee, K. T. \& Craighead, H. G. (2005). Lab Chip, 5, 337-343.

Steigert, J., Grumann, M., Brenner, T., Riegger, L., Harter, J., Zengerle, R. \& Ducrée, J. (2006). Lab Chip, 6, 1040-1044.

Thorsen, T., Roberts, R. W., Arnold, F. H. \& Quake, S. R. (2001). Phys. Rev. Lett. 86, 4163-4166.

Toft, K. N., Vestergaard, B., Nielsen, S. S., Snakenborg, D., Jeppesen, M. G., Jacobsen, J. K., Arleth, L. \& Kutter, J. P. (2008). Anal. Chem. 80, 3648-3654.
Uzawa, T., Akiyama, S., Kimura, T., Takahashi, S., Ishimori, K., Morishima, I. \& Fujisawa, T. (2004). Proc. Natl Acad. Sci. USA, 101, 1171-1176.

Wadsten-Hindrichsen, P., Bender, J., Unga, J. \& Engström, S. (2007). J. Colloid Interface Sci. 315, 701-713.

Weinhausen, B. \& Köster, S. (2013). Lab Chip, 13, 212-215.

Werdich, A. A., Lima, E. A., Ivanov, B., Ges, I., Anderson, M. E., Wikswo, J. P. \& Baudenbacher, F. J. (2004). Lab Chip, 4, 357362.

Wheeler, A. R., Throndset, W. R., Whelan, R. J., Leach, A. M., Zare, R. N., Liao, Y. H., Farrell, K., Manger, I. D. \& Daridon, A. (2003). Anal. Chem. 75, 3581-3586.

Whitesides, G. M. (2006). Nature, 442, 368-373.

Wibroe, P. P., Azmi, I. D. M., Nilsson, C., Yaghmur, A. \& Moghimi, S. M. (2015). Nanomed. Nanotechnol. Biol. Med. 11, 1909-1914.

Yaghmur, A. \& Glatter, O. (2009). Adv. Colloid Interface Sci. 147148, 333-342.

Yaghmur, A., Laggner, P., Almgren, M. \& Rappolt, M. (2008). PLoS One, 3, e3747.

Yaghmur, A., Laggner, P., Sartori, B. \& Rappolt, M. (2008). PLoS One, 3, e2072.

Yaghmur, A., Larsen, S. W., Schmitt, M., Østergaard, J., Larsen, C., Jensen, H., Urtti, A. \& Rappolt, M. (2011). Soft Matter, 7, 82918295 .

Yaghmur, A., Paasonen, L., Yliperttula, M., Urtti, A. \& Rappolt, M. (2010). J. Phys. Chem. Lett. 1, 962-966.

Yaghmur, A. \& Rappolt, M. (2012). Eur. Biophys. J. 41, 831-840.

Yaghmur, A., Sartori, B. \& Rappolt, M. (2011). Phys. Chem. Chem. Phys. 13, 3115-3125.

Zilio, C., Bernardi, A., Palmioli, A., Salina, M., Tagliabue, G., Buscaglia, M., Consonni, R. \& Chiari, M. (2015). Sens. Actuators B Chem. 215, 412-420. 